\title{
High-Risk Features of Delayed Clinical Progression in Cerebral Venous Thrombosis: A Proposed Prediction Score for Early Intervention
}

\author{
Saif A. Bushnaq ${ }^{a}$ Fares Qeadan ${ }^{c}$ Tapan Thacker ${ }^{a}$ Mohammad Abbas ${ }^{b}$ \\ Andrew P. Carlson ${ }^{b}$ \\ aDepartment of Neurology, University of New Mexico School of Medicine, Albuquerque, \\ NM, USA; ${ }^{b}$ Department of Neurosurgery, University of New Mexico School of Medicine, \\ Albuquerque, NM, USA; ${ }^{\circ}$ Clinical Translational Science Center, University of New Mexico \\ School of Medicine, Albuquerque, NM, USA
}

\section{Keywords}

Anticoagulants · Cerebral venous thrombosis - Dural sinus thrombosis · Mechanical thrombectomy · Thrombolysis

\begin{abstract}
Background: Anticoagulation is the mainstay treatment for cerebral venous thrombosis (CVT). A subset of patients might deteriorate despite anticoagulation, and in such cases, endovascular therapy is recommended. Methods: A retrospective review was performed on subjects with CVT from January 2005 to October 2016. The primary outcome was clinical deterioration. Bivariate analysis, multiple logistic regression modeling, and linear discriminant analysis were used to determine a predictive model for deterioration; the results from these models were used to construct a CVT score in order to measure the individual likelihood of deterioration. Results: We identified 147 subjects with CVT. The majority were treated with anticoagulation ( $n=109,74.15 \%)$; 38 (25.85\%) were found to have deterioration, 12 (8.16\%) of whom underwent endovascular intervention. The most important risk factors of deterioration, per bivariate analysis, included decreased level of consciousness (odds ratio [OR] $=5.76 ; 95 \%$ confidence interval $[\mathrm{Cl}] 2.59-12.77)$ and papilledema $(\mathrm{OR}=4.52 ; 95 \% \mathrm{Cl} 1.55-13.18)$. The final multivariable model also included CVT location score (number of sinuses involved), oral contraceptive pill use, sodium level, platelet count, and seizure activity on presentation. This model had a predictive ability to identify deterioration of $83.2 \%$, with a sensitivity of $71.4 \%$ and a specificity of $76.2 \%$. Patients with a CVT score of $\geq 5$ have at least $50 \%$ chance of deterioration. Conclusions: Decreased mental status, seizure activity, papilledema, number of in-
\end{abstract}


volved sinuses, as well as sodium level and platelet count are the most important factors in predicting deterioration after CVT. This group may represent a subset of patients in whom early endovascular therapy may be considered.

(c) 2018 S. Karger AG, Basel

\section{Introduction}

Cerebral venous thrombosis (CVT) is an unusual form of stroke, with extremely heterogeneous presentation due to significant variability in both the venous architecture and the extent of thrombosis, which can range from headache and elevated intracranial pressure to venous infarction and hemorrhage [1]. It is estimated to constitute approximately $0.5-1 \%$ of strokes and usually affects the younger population compared to arterial stroke [2]. The risk factors for CVT can be transient or permanent, including central nervous system or systemic infections, head trauma, drugs with prothrombotic action, genetic conditions, and malignancy [3]. CVT is more common in females taking oral contraceptive pills (OCPs) and in pregnancy [4]. Approximately 13\% of patients in the International Study on Cerebral Vein and Dural Sinus Thrombosis (ISCVT) had poor neurological outcomes, in terms of disability and death, even after treatment with anticoagulation. In the VENOPORT study [5], cerebral edema with or without seizures and sudden cardiopulmonary arrest were the major causes of death.

Endovascular therapy is currently used in a subset of patients who clinically or radiographically deteriorate despite anticoagulation, and it is considered an effective salvage option with a reasonable safety profile, but not supported by high-level evidence $[4,6,7]$. It is intuitive to hypothesize that earlier intervention is more likely to be technically efficacious since there is less fibrin cross-linking in the clot. At the delayed interval, scarring and mechanical stenosis due to inflammatory changes may make the thrombus unamenable to simple thrombolysis or thrombectomy and may require adjunctive procedures such as sinus stenting $[8,9]$. If this high-risk group can be targeted for early intervention, this intervention may be both more effective and avoid permanent neurological injury.

We sought to identify patients who not only had a poor outcome (as has been previously well published $[1,6])$, but those patients with CVT who went on to have clinical deterioration. We then wanted to determine whether there were identifiable features at the time of presentation that could predict a high-risk group in which early endovascular therapy might be considered.

\section{Methods}

This study was conducted at University of New Mexico Hospitals. A retrospective chart review was performed at our institution to identify subjects with CVT from January 2005 to October 2016. Patients were identified using International Classification of Diseases, 9th revision codes. The diagnosis of CVT was established by computed tomography venography, magnetic resonance venography, or catheter-based angiography. We collected extensive baseline clinical, laboratory, and radiographic data on these subjects from the time of initial admission. Clinical risk factors included altered mental status, Glasgow Coma Scale (GCS) score, seizure activity on presentation, papilledema, and OCP use. Laboratory factors included admission complete blood count, comprehensive metabolic panel, liver function tests, lactate, urine drug screen, and hypercoagulable panel. Radiographic data included location of thrombosis, number of sinus segments involved, ischemic stroke, and presence of hemorrhage. From these data, composite risk factors were also constructed to group similar findings. The first composite risk factor was decreased level of consciousness (LOC) (GCS score $<15$, diagnosis of altered mental status at admission, or documented encephalopathy). To standardize the location of thrombus, one point was assigned to each sinus, including superior sagittal sinus, transverse sinus, sigmoid sinus, intracranial jugular vein, straight sinus, deep venous system, or cortical vein. Each location was analyzed both independently and as a composite score (location score). 
Table 1. Patients characteristics

\begin{tabular}{|c|c|c|c|}
\hline Characteristics & $\begin{array}{l}\text { No deterioration } \\
n / N(\%)\end{array}$ & $\begin{array}{l}\text { Deterioration } \\
n / N(\%)\end{array}$ & $\begin{array}{l}\text { Total } \\
n / N(\%)\end{array}$ \\
\hline \multicolumn{4}{|l|}{ Age } \\
\hline$\leq 18$ years & $24 / 109(22.02)$ & $10 / 38(26.32)$ & $34 / 147(23.13)$ \\
\hline$>18$ years & $85 / 109$ (77.98) & $28 / 38(73.68)$ & $113 / 147(76.87)$ \\
\hline \multicolumn{4}{|l|}{ Sex } \\
\hline Female & $60 / 107$ (56.07) & $19 / 36(52.78)$ & $79 / 143(55.24)$ \\
\hline Male & $47 / 107$ (43.93) & $17 / 36(47.22)$ & $64 / 143(44.76)$ \\
\hline \multicolumn{4}{|l|}{ Glasgow Coma Scale score ${ }^{1}$} \\
\hline 15 & $87 / 105(82.86)$ & $18 / 36(50.00)$ & $105 / 141(74.47)$ \\
\hline $3-14$ & $18 / 105(17.14)$ & $18 / 36(50.00)$ & $36 / 141(25.53)$ \\
\hline \multicolumn{4}{|l|}{ Glasgow Coma Scale score } \\
\hline$>8$ & 98/105 (93.33) & $30 / 36(83.33)$ & $128 / 141(90.78)$ \\
\hline$\leq 8$ & $7 / 105(6.67)$ & $6 / 36(16.67)$ & $13 / 141(9.22)$ \\
\hline \multicolumn{4}{|c|}{ Hemorrhage on initial head computed tomography } \\
\hline Yes & $36 / 109(33.03)$ & $18 / 38(47.37)$ & $54 / 147(36.73)$ \\
\hline No & $73 / 109(66.97)$ & $20 / 38(52.63)$ & $93 / 147(63.27)$ \\
\hline \multicolumn{4}{|c|}{ Ischemic infarction on initial imaging } \\
\hline Yes & $15 / 107(14.02)$ & $10 / 38(26.32)$ & $25 / 145(17.24)$ \\
\hline No & $92 / 107(85.98)$ & $28 / 38(73.68)$ & $120 / 145(82.76)$ \\
\hline \multicolumn{4}{|l|}{ Seizure activity ${ }^{1}$} \\
\hline Yes & $11 / 109(10.09)$ & $10 / 38(26.32)$ & 21/147 (14.29) \\
\hline No & $98 / 109(89.91)$ & $28 / 38(73.68)$ & $126 / 147(85.71)$ \\
\hline \multicolumn{4}{|l|}{ Papilledema $^{1}$} \\
\hline Yes & $7 / 109(6.42)$ & 9/38 (23.68) & $16 / 147(10.88)$ \\
\hline No & $102 / 109(93.58)$ & $29 / 38(76.32)$ & $131 / 147(89.12)$ \\
\hline \multicolumn{4}{|c|}{ Location score (number of sinuses involved) ${ }^{1}$} \\
\hline$>3$ & $14 / 108(12.96)$ & $13 / 37(35.14)$ & $27 / 145(18.62)$ \\
\hline$\leq 3$ & $94 / 108(87.04)$ & $24 / 37(64.86)$ & $118 / 145(81.38)$ \\
\hline \multicolumn{4}{|l|}{ Oral contraceptive pill use } \\
\hline Yes & $11 / 109(10.09)$ & $1 / 38(2.63)$ & $12 / 147(8.16)$ \\
\hline No & $98 / 109(89.91)$ & $37 / 38(97.37)$ & $135 / 147(91.84)$ \\
\hline Mean age, years & 35.65 & 35.32 & 35.56 \\
\hline Mean sodium level, mEq/L & 140.28 & 138.71 & 139.88 \\
\hline Mean platelet count, $\times 10^{9} / \mathrm{L}$ & 257.75 & 226.17 & 249.51 \\
\hline
\end{tabular}

${ }^{1}$ Statistically significant according to the $\chi^{2}$ test for independence at the $5 \%$ significance level.

We defined our primary outcome as a binary composite measure of delayed deterioration which was constructed based on symptomatic worsening due to CVT at follow-up visits, death, repeat emergency room visits after discharge for CVT-related complaints, or need for endovascular intervention. Symptomatic worsening due to CVT included new neurological deficits such as focal weakness or cranial nerve abnormalities, worsening mental status or decrement in GCS score, new or worsening bleeding on neuroimages like midline shift, worsening edema, or mass effect.

We performed bivariate analysis using logistic regression for each independent variable to identify potential risk factors for deterioration. We then performed multiple logistic regression modeling and linear discriminant analysis to determine a predictive model for deterioration using the best subset method which gives maximal accuracy while avoiding overfitting $[10,11]$. We used the area under the receiver operating characteristic (ROC) curve as a measure for classification accuracy. The independent variables from this predictive model were used to construct a risk stratification scale, named the CVT score, to give a rough estimate of the individual likelihood of deterioration. Sensitivity and specificity analysis for the predictive model was done. For all significant continuous predictors for deterioration, we identified optimal thresholds by minimizing the Euclidean distance of sensitivity and specificity from the unitary point $(1,1)[12]$. Statistical analysis was performed using SAS version 9.4 (SAS Institute Inc., Cary, NC, USA). 
Table 2. Association between anticoagulation and deterioration

\begin{tabular}{llll}
\hline Anticoagulation & Deterioration & \\
\cline { 2 - 3 } & no & yes & total \\
\hline No & & & \\
$\quad$ Frequency & 25 & 10 & 35 \\
$\quad$ Row percentage & 71.43 & 28.57 & 24.31 \\
$\quad$ Column percentage & 23.36 & 27.03 & \\
\hline Yes & & & 75.69 \\
$\quad$ Frequency & 75.23 & 27 & \\
$\quad$ Row percentage & 76.64 & 24.77 & 144 \\
Column percentage & & 72.97 & 100.00 \\
\hline Total & 107 & 37 & \\
$\quad$ Frequency & 74.31 & 25.69 & \\
$\quad$ Percentage & & & \\
\hline
\end{tabular}

Anticoagulation by deterioration: $p=0.6544$ (statistically not significant according to the $\chi^{2}$ test for independence at the $5 \%$ significance level).

\section{Results}

\section{General Characteristics}

We identified 147 patients who met the inclusion criteria. Most patients were aged $>18$ years $(76.87 \%)$ and females $(55.24 \%)$. Headache was the most common symptom $(n=98$, $66.67 \%)$, followed by nausea or vomiting ( $n=58,39.46 \%)$, focal weakness ( $n=33,22.45 \%)$, seizure $(n=21,14.29 \%)$, and papilledema $(n=16,10.88 \%)$. The mean time to presentation was 8.9 days. The majority of patients were treated with anticoagulation $(n=109,74.15 \%)$. Urine toxicology screen was positive in 14 patients with benzodiazepines and/or amphetamine, though this was not routinely measured on all patients. The patient characteristics are summarized in Table 1.

Hypercoagulable labs were tested in $81 / 147$ patients, and despite many patients undergoing early testing, positive results were very uncommon. Three out of 25 patients tested had elevated plasma homocystine. Twenty-one out of 81 patients tested had positive lupus-like inhibitor. Factor 5 Leiden mutation was found in 3 out of 75 patients tested. Because of the relatively smaller numbers, these factors were ultimately not considered in the final model.

The location of CVT was as follows: transverse sinus involvement was most common in 100 patients (68.03\%), sigmoid sinus in 94 (63.95\%), superior sagittal sinus in $53(36.05 \%)$, bilateral transverse sinus in 18 (12.24\%), bilateral sigmoid sinus in $4(2.72 \%)$, internal jugular vein in 31 (21.08\%), straight sinus in 17 (11.56\%), deep venous system in 12 (8.16\%), and cortical vein in 13 (8.84\%).

Out of 147 patients, $38(25.85 \%)$ were found to have deterioration, $12(8.16 \%)$ of whom required endovascular intervention. The other cases of deterioration were due to clinical worsening [12], death [10], and repeat emergency room visits [13]. Out of 35 patients who had no anticoagulation, 10 deteriorated, but this was not significant (odds ratio [OR] = 1.215; 95\% confidence interval [CI] 0.518-2.850). The association between anticoagulation and deterioration is presented in Table 2. Reasons for no anticoagulation included venous hemorrhage on initial images (3 patients), trauma (11 patients), concurrent central nervous system infection ( 2 patients), gastrointestinal bleeding ( 2 patients), arteriovenous fistula causing 

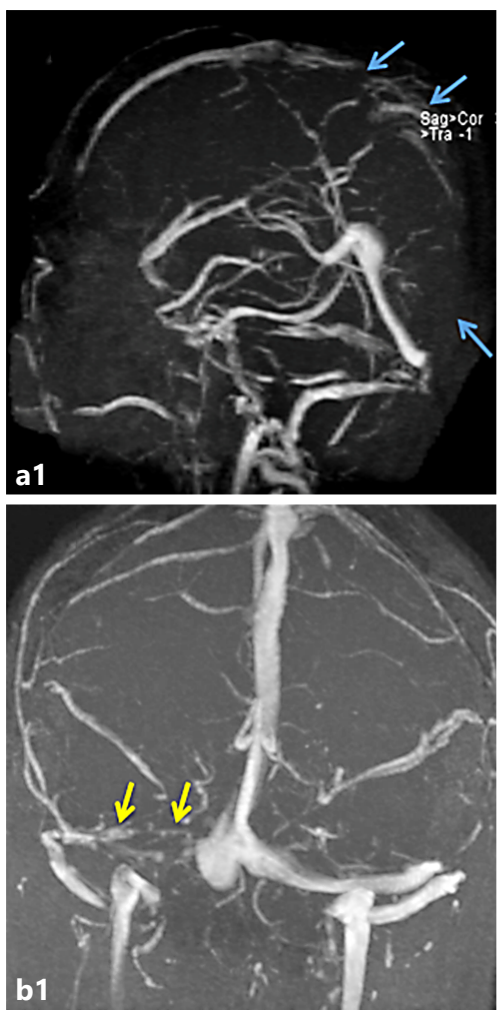
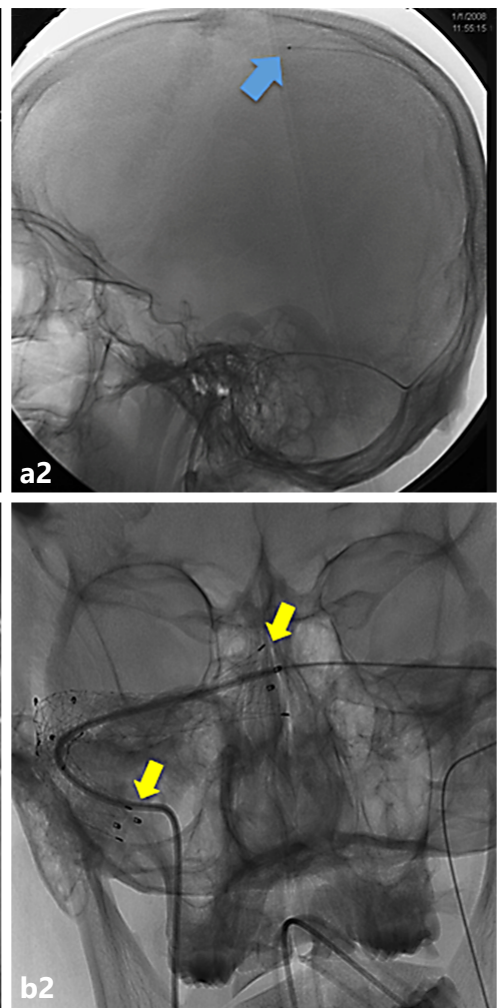
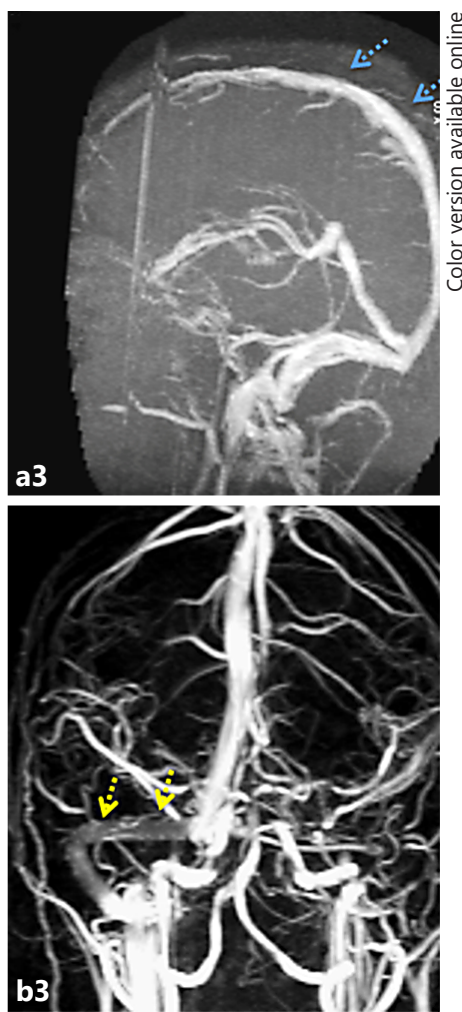

Fig. 1. Two patients who underwent endovascular therapy. a A patient with superior sagittal sinus thrombosis on magnetic resonance venography (a1, blue arrows) which extended through the right transverse, sigmoid, and jugular. Due to the extensive thrombosis, the patient was taken for early thrombolysis (a2, microcatheter in the superior sagittal sinus, wide blue arrow.) Tissue plasminogen activator was infused for 2 days with daily angiograms to assess progress. Magnetic resonance venography 3 days later showed near complete recanalization of the entire superior sagittal, transverse, sigmoid, and jugular (a3, dotted blue arrows). b A patient with right transverse and sigmoid thrombosis (b1, yellow arrows.) Despite anticoagulation, the patient developed severe papilledema and bilateral sixth nerve palsies 4 weeks later. Attempted thrombolysis and balloon thrombectomy had been unsuccessful and a transverse sinus stent was placed (b2, wide yellow arrows). The patient's papilledema and sixth nerve palsies reversed rapidly, and 1-year followup magnetic resonance venography showed wide patency through the stents (b3, dotted yellow arrows).

sinus thrombosis (1 patient), as well as underlying malignancy and decision for hospice care and no further treatment ( 4 patients). Outcomes for the 12 patients with clinical worsening included 6 patients with mild deficits (Rankin scores 1 and 2) and 4 patients with moderate to severe deficits (Rankin scores 3 and 4). As regards mortality, 6 patients were attributed to CVT. One patient died from acute myeloid leukemia, the other patient died due to hemorrhagic shock form gastrointestinal bleeding. The median time of hospitalization was 9.76 days. Fifteen patients developed $\geq 1$ seizure during hospitalization.

\section{Patients Treated with Endovascular Therapy}

We treated 12 patients with endovascular therapy. Most of them (9/12) were treated with anticoagulation on the day of the diagnosis or on the next day. Indications for endovascular intervention were primarily uncontrolled elevated intracranial pressure with papilledema as well as large venous infarct with hemorrhagic transformation and extensive thrombosis with poor clinical exam. Three patients had venous infarcts and hemorrhagic 
Table 3. Bivariate analysis (provides unadjusted ORs)

\begin{tabular}{|c|c|c|}
\hline Presenting symptoms and risk factors & OR $(95 \% \mathrm{CI})$ & $p$ value \\
\hline Decreased level of consciousness & $5.76(2.60-12.77)$ & $<0.0001^{*}$ \\
\hline Initial Glasgow Coma Scale score & $4.83(2.11-11.05)$ & $0.0002^{*}$ \\
\hline Papilledema & $4.52(1.55-13.19)$ & $0.006^{*}$ \\
\hline Altered mental status & $3.96(1.80-8.71)$ & $0.001^{*}$ \\
\hline Bilateral transverse sinus thrombosis & $3.45(1.25-9.49)$ & $0.02^{*}$ \\
\hline Seizure activity & $3.18(1.23-8.26)$ & $0.02 *$ \\
\hline Unilateral internal jugular thrombosis & $2.19(0.94-5.09)$ & 0.07 \\
\hline Ischemic infarct on neuroimages & $2.19(0.89-5.42)$ & 0.09 \\
\hline Bleeding on initial neuroimages & $1.83(0.86-3.90)$ & 0.12 \\
\hline Location score (number of sinuses involved) & $1.35(1.03-1.76)$ & $0.03^{*}$ \\
\hline Nausea/vomiting & $1.34(0.64-2.84)$ & 0.44 \\
\hline Focal weakness & $1.34(0.57-3.14)$ & 0.51 \\
\hline Cortical vein thrombosis & $1.31(0.38-4.52)$ & 0.68 \\
\hline Superior sagittal sinus thrombosis & $1.26(0.58-2.70)$ & 0.56 \\
\hline Elevated total bilirubin & $1.12(0.87-1.44)$ & 0.38 \\
\hline Unilateral sagittal sinus thrombosis & $1.12(0.51-2.42)$ & 0.78 \\
\hline Elevated potassium & $1.02(0.95-1.10)$ & 0.60 \\
\hline Elevated creatinine & $1.01(0.62-1.64)$ & 0.98 \\
\hline Protein S deficiency & $1.00(1.00-1.02)$ & 0.84 \\
\hline Platelet count & $1.00(0.99-1.00)$ & 0.13 \\
\hline Elevated blood urea nitrogen & $1.00(0.97-1.02)$ & 0.74 \\
\hline Elevated lactate & $0.99(0.77-1.28)$ & 0.95 \\
\hline Deep venous sinus thrombosis & $0.95(0.24-3.72)$ & 0.94 \\
\hline Sodium level & $0.92(0.82-1.03)$ & 0.16 \\
\hline Abnormal hypercoagulable panel & $0.89(0.35-2.24)$ & 0.80 \\
\hline Sex & $0.88(0.41-1.87)$ & 0.73 \\
\hline Straight sinus thrombosis & $0.87(0.27-2.85)$ & 0.82 \\
\hline Headache & $0.81(0.38-1.76)$ & 0.59 \\
\hline Decreased visual acuity & $0.77(0.26-2.23)$ & 0.62 \\
\hline Unilateral transverse sinus thrombosis & $0.74(0.34-1.62)$ & 0.46 \\
\hline Cranial sixth nerve palsy & $0.69(0.12-3.90)$ & 0.67 \\
\hline Albumin & $0.48(0.23-1.01)$ & 0.05 \\
\hline Oral contraceptive pill use & $0.24(0.03-1.93)$ & 0.18 \\
\hline
\end{tabular}

CI, confidence interval; OR, odds ratio. * Statistically significant $(p<0.05)$.

transformation before endovascular treatment. One patient had thrombosis of the deep cerebral venous system and ultimately died despite initial anticoagulation and chemical thrombolysis with tissue plasminogen activator due to intraventricular hemorrhage and hydrocephalus.

Four patients out of 12 had mechanical thrombectomy (aspiration, stent retriever thrombectomy, or balloon thrombectomy), 5 patients had intra-arterial thrombolysis, 1 patient had thrombolysis with thrombectomy, 1 patient underwent thrombectomy with stenting, and 1 patient had stenting primarily. Seven patients had complete or near complete recanalization, 2 patients had partial recanalization, and 2 patients had no recanalization. Eight patients were discharged home (recovered or with minimal residual symptoms with Rankin scores 0 or 1), 2 patients had moderate or severe deficits (Rankin scores 3 and 4), and 2 patients died. One death was attributed to trauma and coma prior to intervention, the other was due to extensive deep sinus thrombosis (see Fig. 1 for representative cases of early and late intervention). 
Fig. 2. a ORs with $95 \%$ Wald confidence limits from the final multivariable model. b ROC curve for the model. LOC, level of consciousness; OCPs, oral contraceptive pills; OR, odds ratio; ROC, receiver operating characteristic.

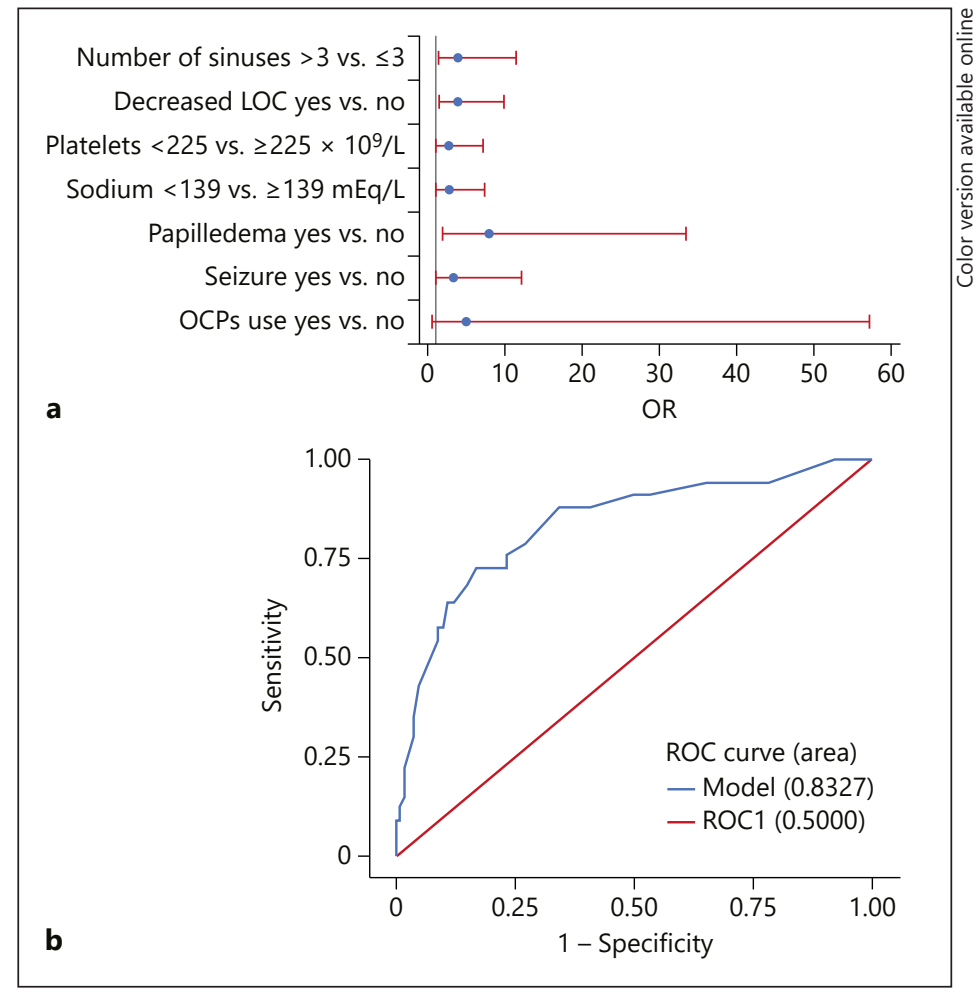

\section{Risk Factors for Deterioration}

The risk factors for deterioration on bivariate analysis are summarized in Table 3. Significant factors included decreased LOC (OR $=5.76$; 95\% CI 2.60-12.77), initial GCS score (OR = 4.83; 95\% CI 2.11-11.05), papilledema (OR $=4.52$; 95\% CI 1.55-13.19), seizure activity on presentation $(\mathrm{OR}=3.18$; 95\% CI 1.23-8.26), bilateral transverse sinus thrombosis $(\mathrm{OR}=3.45$; 95\% CI 1.25-9.48), altered mental status (OR $=3.96$; 95\% CI 1.80-8.71), and location score (OR = 1.35; 95\% CI 1.02-1.76).

We tested multiple predictive models of deterioration using multivariable logistic regression. For practical use of these models, we calculated the optimum threshold for the continuous variables and then converted them to binary for the multivariable analysis. The selected final model (Fig. 2) included the following seven predictors: number of sinuses $>3$ $(\mathrm{OR}=3.80 ; 95 \% \mathrm{CI} 1.27-11.39)$, decreased LOC (OR = 3.73; 95\% CI 1.43-9.76), platelet count $<225 \times 10^{9} / \mathrm{L}(\mathrm{OR}=2.61 ; 95 \% \mathrm{CI} 0.96-7.09)$, sodium level $<139 \mathrm{mEq} / \mathrm{L}(\mathrm{OR}=2.59 ; 95 \% \mathrm{CI}$ 0.93-7.19), papilledema (OR $=7.88 ; 95 \% \mathrm{CI} 1.87-33.33$ ), seizure activity (OR $=3.16$; 95\% CI $0.83-11.99$ ), and no OCP use (OR $=4.90 ; 95 \%$ CI 0.42-57.19). Though not each of these was individually significant in the model, the combination resulted in a model with good accuracy and discrimination power with $83.2 \%$ area under the ROC curve, which decreased with attempted stepwise reduction of variables. A post hoc analysis of linear discrimination confirmed that the final model had a sensitivity of $72.73 \%$, a specificity of $78.79 \%$, a positive predictive value of $53.33 \%$, and a negative predictive value of $89.66 \%$.

To construct the risk stratification scale (the CVT score) for calculating individual probabilities of deterioration, ORs were calculated for each of the binary predictors from the final model. We assigned one point to ORs around 2.5 (the lowest OR in the model) and multiple points based on multiplicities of 2.5 (see Fig. 3 for the detailed assignment). Ten possible points were assigned for the proposed model. One point was assigned for $>3$ sinuses involved, 


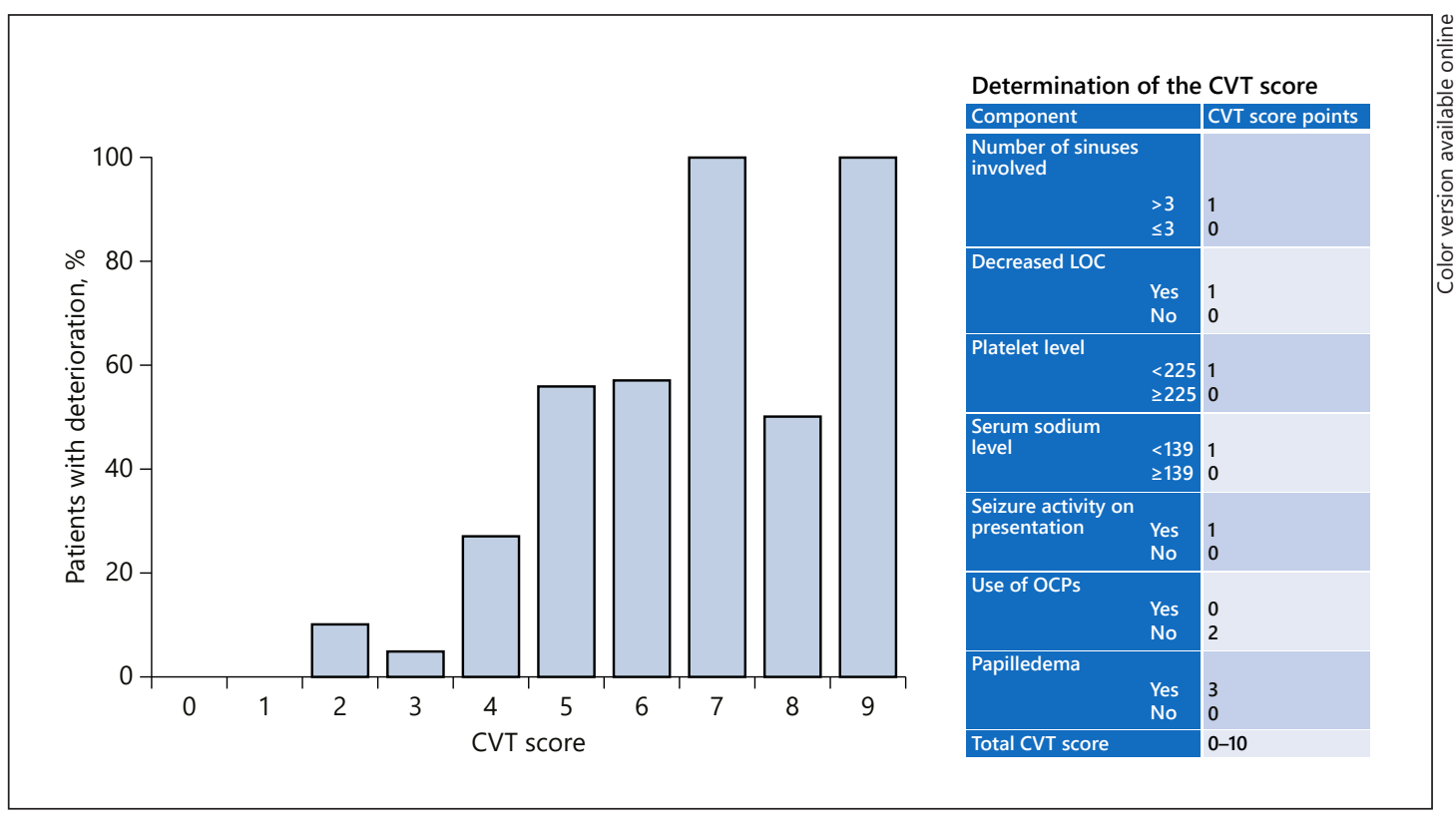

Fig. 3. CVT score to predict early deterioration. The proposed score (right) was applied to our data set, revealing these percentages of patients with deterioration. Note that a score of $\geq 5$ results in a $\geq 50 \%$ chance of deterioration versus a much lower risk with a lower score. Though this is a 10-point scale, none of our patients had a score of 10, so none was omitted from this figure. CVT, cerebral venous thrombosis; LOC, level of consciousness; OCPs, oral contraceptive pills.

decreased LOC, platelets count $<225 \times 10^{9} / \mathrm{L}$, serum sodium level $<139 \mathrm{mEq} / \mathrm{L}$, and seizure activity on presentation. Two points were assigned for no OCP use, and three points were assigned for the presence of papilledema. The individual likelihood of deterioration by CVT score was then calculated based on the existing data set (Fig. 3). This analysis showed that patients with a CVT score of $\geq 5$ have at least $50 \%$ chance of deterioration, whereas scores of $<5$ demonstrate very low risk of deterioration. A CVT score of 5 seemed to be the optimal prediction threshold to stratify patients for potential to need earlier intervention based on our data.

\section{Discussion}

Although CVT is an uncommon stroke in the general population, it can be potentially lifethreatening and disproportionately affects younger patients. In the ISCVT, $4.3 \%$ of patients died during the acute phase of CVT and 3.4\% within 30 days from symptom onset [1]. The incidence of CVT has been reported to be as high as 1.32 per 100000 person-years, which is higher than previously published [14]. This incidence may be increased at higher elevation such as ours (mean 5,700 feet above sea level) in New Mexico due to relative thrombophilia [15].

CVT presentation is quite variable. Headache, usually unilateral, is the most common presenting symptom in 70-90\% of patients and can be the sole complaint [16]. In our study, headache was also the most common symptom, reported in $66.67 \%$. Other commonly reported symptoms include focal neurological deficit in $40.8 \%$, seizure in $30.2 \%$, and altered mental status in $40.2 \%$ [4]. The superior sagittal sinus was the most common location $(72.2 \%$ ) 
in that series. In comparison, we found focal weakness in $22.45 \%$ and seizure in $14.29 \%$, while transverse sinus involvement was the most common location $(68 \%)$ in our series. Mortality occurs in the range of 3-5\% [13]. In our cohort, the deaths of 6/147 patients (4.08\%) were related to CVT.

The European Federation of Neurological Societies guidelines on the treatment of CVT [17] recommend that patients with CVT and no contraindications for anticoagulation should be treated with anticoagulation. The 2011 American Heart Association/American Stroke Association guidelines state that anticoagulation is reasonable for patients with acute CVT, even in selected patients with intracranial hemorrhage [6]. In our cohort, 109/147 (74.15\%) patients were started on anticoagulation. Though we did not investigate the specific reasons for no anticoagulation, this is done in cases where there is a large amount of hemorrhage, need for surgical intervention, or perceived benign nature of the thrombosis by the treating team.

Endovascular techniques for CVT have evolved and new devices have been introduced over the last decade. Different techniques include direct catheter thrombolysis, balloonassisted thrombectomy, rheolytic catheter thrombectomy, aspiration thrombectomy, and stent retriever thrombectomy [4]. Multimodality treatment is often required, though several centers have reported success with suction aspiration [18]. In our cohort, multimodality treatment was used in 6 patients ( 2 patients had complete recanalization, 2 had partial recanalization, 1 had no recanalization, and 1 died with extensive deep venous sinus thrombosis). Five patients underwent only intra-arterial thrombolysis, and 1 patient underwent stenting.

In a recent systematic review of 17 studies (235 patients) which evaluated the use of endovascular therapy for CVT [4], the indications for endovascular therapy included failure of systemic anticoagulation, extensive clot burden, cerebral edema, altered mental status, and progressively worsening neurological symptoms. Eighty-two percent $(155 / 189)$ of their patients were started on anticoagulation prior to intervention. Postoperative complete radiographic resolution of CVT was achieved in $69.0 \%$ (147/213) of cases, while partial resolution was achieved in 26.3\% (56/213). The Thrombolysis or Anticoagulation for Cerebral Venous Thrombosis trial (NCT01204333) is a closed multicenter, prospective trial comparing the safety and efficacy of endovascular therapy with medical therapy in high-risk patients which unfortunately did not reach recruitment goals to continue enrollment. The investigators defined high risk factors as decreased mental status, hemorrhage, or deep venous thrombosis. In our data, of these only decreased mental status was a significant predictor of deterioration. Our contention is that it is not simply the poor outcome with CVT group that should be targeted, but also patients who start out with a relatively benign exam and then deteriorate. Many patients with poor outcome have a severe problem at admission (such as thalamic infarction with deep thrombosis) and so may not be the optimal group to target for intervention. The concept of early intervention in certain patients, however, does make sense in that delaying thrombectomy until deterioration or failure of anticoagulation has the unwanted effects of (1) allowing the clot to become fibrous and less amenable to simple thrombectomy and (2) potentially allowing for new permanent neurological deficits to develop. The above systematic review also supports this role for earlier intervention in terms of improved odds of good outcome in patients treated early (OR $=0.12$; 95\% CI 0.02-0.86; $p=0.03$ ).

Based on the model we developed, decreased LOC at admission, platelet count $<225 \times$ $10^{9} / \mathrm{L}$, sodium level $<139 \mathrm{mEq} / \mathrm{L}$, presence of seizure activity, absence of OCP use, and number of sinuses involved $>3$ all were important factors in predicting deterioration. Though some of these factors were not initially expected to be significant, we think that they make sense physiologically. Thrombocytosis and hypernatremia are likely reflective of dehydration (a reversible risk factor), and the use of OCPs is similarly reversible, so these would result in less chance of progression. Decreased mental status, more segmental sinuses involved, and 
seizure are all likely reflective of more severe disease. A CVT score of $\geq 5$ points seemed to be highly predictive of a greater chance of poor outcome. This is a simple scoring system that uses easily accessible data from admission to determine who should be considered for intervention. Prospective assessment is required for refinement and validation of this score; nonetheless, we feel that it at least offers preliminary guidance in decision-making if thrombectomy is being considered.

The limitations of our study include the use of a retrospective cohort with relatively small sample size and the lack of standardized criteria for endovascular therapy. The composite outcome of deterioration we used was intended to capture a variety of patients with deterioration, not just patients with poor outcome, but also makes for a somewhat less defined group compared to a simple poor modified Rankin Scale score or another ordinal scale of outcome. Our goal for doing this was specifically to target patients who have a higher likelihood of salvage, since many patients with poor outcome likely have such a poor prognosis from the onset and may not have a significant opportunity for intervention. It is also possible that some patients with deterioration were missed because there were no specific follow-up requirements, but since our institution is the only tertiary referral center in New Mexico, most patients with delayed deterioration will return to our institution. Finally, there may be other risk factors, particularly within the hypercoagulable panel, that may also be relevant, but as many of these were not routinely obtained at admission, we did not have enough numbers to analyze them as specific risk factors. That said, our model provided a surprisingly high predictive value with the data we were able to incorporate.

\section{Conclusions}

Many patients with CVT (up to 25\%) are at risk of deterioration despite anticoagulation. Decreased mental status, seizure activity, papilledema, number of involved sinuses, low serum sodium level, and low platelet count are the most important combination of risk factors in predicting deterioration after CVT. We present a prediction score based on these clinical, radiographic, and laboratory data at admission which performed well in our retrospective cohort to predict the status of these patients. We hypothesize that this subgroup would be the optimal target for early venous thrombectomy or thrombolysis. Prospective validation and ultimately trials are required to confirm this hypothesis.

\section{Statement of Ethics}

Ethics approval was obtained from the institutional review board of the University of New Mexico School of Medicine.

\section{Disclosure Statement}

The authors have no conflicts of interest to disclose. This research received no specific grant from any funding agency in the public, commercial, or not-for-profit sectors. 


\section{Interventional Neurology}

\begin{tabular}{l|l}
\hline Intervent Neurol 2018;7:297-307 \\
\hline DOI: 10.1159/000487960 & $\begin{array}{l}\text { (c) 2018 S. Karger AG, Basel } \\
\text { www.karger.com/ine }\end{array}$ \\
\hline
\end{tabular}

Bushnaq et al.: A Proposed Cerebral Venous Thrombosis Score

\section{References}

1 Ferro JM, Canhão P, Stam J, Bousser MG, Barinagarrementeria F; ISCVT Investigators: Prognosis of cerebral vein and dural sinus thrombosis: results of the International Study on Cerebral Vein and Dural Sinus Thrombosis (ISCVT). Stroke 2004;35:664-670.

2 Stam J: Thrombosis of the cerebral veins and sinuses. N Engl J Med 2005;352:1791-1798.

-3 Ferro JM, Canhão P: Cerebral venous sinus thrombosis: update on diagnosis and management. Curr Cardiol Rep 2014;16:523.

4 Ilyas A, Chen CJ, Raper DM, Ding D, Buell T, Mastorakos P, Liu KC: Endovascular mechanical thrombectomy for cerebral venous sinus thrombosis: a systematic review. J Neurointerv Surg 2017;9:1086-1092.

5 Ferro JM, Lopes MG, Rosas MJ, Ferro MA, Fontes J; Cerebral Venous Thrombosis Portuguese Collaborative Study Group: Long-term prognosis of cerebral vein and dural sinus thrombosis. Results of the VENOPORT study. Cerebrovasc Dis 2002;13:272-278.

-6 Saposnik G, Barinagarrementeria F, Brown RD Jr, Bushnell CD, Cucchiara B, Cushman M, deVeber G, Ferro JM, Tsai FY; American Heart Association Stroke Council and the Council on Epidemiology and Prevention: Diagnosis and management of cerebral venous thrombosis: a statement for healthcare professionals from the American Heart Association/American Stroke Association. Stroke 2011;42:1158-1192.

7 Mammen S, Keshava SN, Moses V, Aaron S, Ahmed M, Chiramel GK, Mani SE, Alexander M: Role of penumbra mechanical thrombectomy device in acute dural sinus thrombosis. Indian J Radiol Imaging 2017;27:82-87.

-8 Adachi H, Mineharu Y, Ishikawa T, Imamura H, Yamamoto S, Todo K, Yamagami H, Sakai N: Stenting for acute cerebral venous sinus thrombosis in the superior sagittal sinus. Interv Neuroradiol 2015;21:719-723.

-9 Matsumoto N, Kuramoto Y, Shinoda N, Ueno Y: A case of stenting for acute cerebral venous sinus thrombosis in the superior sagittal sinus. Interv Neuroradiol 2016;22:709-710.

10 King JE: Running a best-subsets logistic regression: an alternative to stepwise methods. Educ Psychol Meas 2003;63:392-403.

11 Vittinghoff E, McCulloch CE: Relaxing the rule of ten events per variable in logistic and Cox regression. Am J Epidemiol 2007;165:710-718.

12 Saeed AI, Qeadan F, Sood A, VanderJagt DJ, Mishra SI, Hill DA, Peikert T, Sopori ML: A novel cytokine profile associated with cancer metastasis to mediastinal and hilar lymph nodes identified using fine needle aspiration biopsy - a pilot study. Cytokine 2017;89:98-104.

$\checkmark 13$ Coutinho JM, Zuurbier SM, Stam J: Declining mortality in cerebral venous thrombosis: a systematic review. Stroke 2014;45:1338-1341.

14 Coutinho JM, Zuurbier SM, Aramideh M, Stam J: The incidence of cerebral venous thrombosis: a cross-sectional study. Stroke 2012;43:3375-3377.

15 Zavanone C, Panebianco M, Yger M, Borden A, Restivo D, Angelini C, Pavone A, Grimod G, Rosso C, Dupont S: Cerebral venous thrombosis at high altitude: a systematic review. Rev Neurol (Paris) 2017;173:189-193.

-16 Wasay M, Kojan S, Dai AI, Bobustuc G, Sheikh Z: Headache in cerebral venous thrombosis: incidence, pattern and location in 200 consecutive patients. J Headache Pain 2010;11:137-139.

17 Einhäupl K, Stam J, Bousser MG, De Bruijn SF, Ferro JM, Martinelli I, Masuhr F; European Federation of Neurological Societies: EFNS guideline on the treatment of cerebral venous and sinus thrombosis in adult patients. Eur J Neurol 2010;17:1229-1235.

-18 Mokin M, Lopes DK, Binning MJ, Veznedaroglu E, Liebman KM, Arthur AS, Doss VT, Levy EI, Siddiqui AH: Endovascular treatment of cerebral venous thrombosis: contemporary multicenter experience. Interv Neuroradiol 2015;21:520-526. 